\title{
Gauge symmetry enhancing-breaking from a Double Field Theory perspective
}

\author{
G. Aldazabal, ${ }^{a, b}$ E. Andrés, ${ }^{a}$ Martín Mayo $^{a}$ and J.A. Rosabal ${ }^{c}$ \\ ${ }^{a}$ G. Física CAB-CNEA and CONICET, Centro Atómico Bariloche, \\ Av. Bustillo 9500, Bariloche, Argentina \\ ${ }^{b}$ Instituto Balseiro, Centro Atómico Bariloche, \\ Av. Bustillo 9500, Bariloche, Argentina \\ ${ }^{c}$ B.W. Lee Center for Fields, Gravity $\&$ Strings, Institute for Basic Sciences, \\ Daejeon 34047, Korea \\ E-mail: aldazaba@cab.cnea.gov.ar, eduardo.c.andres@gmail.com, \\ martin.mayo@ib.edu.ar, arosabal@df.uba.ar
}

ABSTRACT: Gauge symmetry enhancing, at specific points of the compactification space, is a distinguished feature of string theory. In this work we discuss the breaking of such symmetries with tools provided by Double Field Theory (DFT). As a main guiding example we discuss the bosonic string compactified on a circle where, at the self-dual radio the generic $\mathrm{U}(1) \times \mathrm{U}(1)$ gauge symmetry becomes enhanced to $\mathrm{SU}(2) \times \mathrm{SU}(2)$. We show that the enhancing-breaking of the gauge symmetry can be understood through a dependence of gauge structure constants (fluxes in DFT) on moduli. This dependence, in DFT description, is encoded in the generalized tangent frame of the double space. The explicit T-duality invariant formulation provided by DFT proves to be a helpful ingredient. The link with string theory results is discussed and generalizations to generic tori compactifications are addressed.

KEYwords: Bosonic Strings, Flux compactifications, String Duality

ARXIV EPRINT: 1704.04427 


\section{Contents}

1 Introduction $\quad 1$

2 DFT and enhanced gauge symmetries $\quad 5$

2.1 Enhanced gauge symmetry on the circle 8

3 Enhancing-breaking of gauge symmetries for generic toroidal compactifications $\quad 12$

$\begin{array}{lll}4 & \text { Summary and outlook } & 17\end{array}$

$\begin{array}{lr}\text { A Vertex operators and enhancing } & 19\end{array}$

$\begin{array}{ll}\text { A.1 Torus example } & 19\end{array}$

B General enhancing groups $\quad 20$

B.1 Vector masses 20

B.2 Scalar masses 21

\section{Introduction}

The extended nature of strings is responsible for several amazing phenomena that are not conceivable from a field theory of point particles. When moving on compact space, besides the expected states associated to KK compact momenta, a string can wind around noncontractible cycles leading to the so-called winding states, with the winding number being an integer counting the number of times that the cycle is wrapped by the string. Quantum states are thus labelled by specific values of KK momenta and windings.

The interplay among winding and momentum modes underlies T-duality, a genuine stringy feature. Such interplay manifests itself by connecting the physics of strings defined on geometrically very different backgrounds. At specific points of moduli of the compact space, states in some combinations of windings and momenta become massless and can give rise to enhanced gauge symmetries (see for instance $[1,2]$ ). The simplest example is provided by the compactification of the bosonic string on a circle of radio $R$. The resulting theory, which contains a $\mathrm{U}(1) \times \mathrm{U}(1)$ gauge group, is equivalent to a string compactified on a circle of radio $\tilde{R}=\frac{\alpha^{\prime}}{R}$ (where $\alpha^{\prime}$ is the string constant) if momenta and winding are exchanged. At the self-dual point $R=\tilde{R}=\sqrt{\alpha^{\prime}}$ the gauge symmetry is enhanced to $\mathrm{SU}(2) \times \mathrm{SU}(2)$.

When the compact space is a $r$ dimensional torus $T^{r}$, characterized by some background moduli (internal metric and anti-symmetric fields), T-duality implies that backgrounds related by the non-compact group $O(r, r, \mathbb{Z})$ are physically equivalent. Generically a richer structure of points of gauge enhancing appears. 
Recall that, from the world sheet point of view, states are created by vertex operators involving both coordinates associated with momentum excitations and dual coordinates associated to winding excitations or, equivalently, to left $(\mathrm{L})$ and right $(\mathrm{R})$ moving coordinates. For generic values of moduli an Abelian symmetry $\mathrm{U}(1)_{L}^{r} \times \mathrm{U}(1)_{R}^{r}$ appears. However, at specific points, the symmetry becomes enhanced to a gauge symmetry $G_{L} \times G_{R}$ where $G_{L(R)}$ are non-Abelian gauge groups of rank $r$. For example, in a two torus $T^{2}$, a generic $(\mathrm{U}(1) \times \mathrm{U}(1))_{L} \times(\mathrm{U}(1) \times \mathrm{U}(1))_{R}$ is enhanced to $\mathrm{SU}(3)_{L} \times \mathrm{SU}(3)_{R}$ or $(\mathrm{SU}(2) \times \mathrm{SU}(2))_{L} \times(\mathrm{SU}(2) \times \mathrm{SU}(2))_{R}$ etc. at different points.

Let us sketch, as motivation of our work, the case of circle compactification at self-dual point. ${ }^{1}$ The effective action in $d$ dimensional space, computed from string theory 3 -point amplitudes [3] reads

$$
\begin{aligned}
S= & \frac{1}{2 \kappa_{d}^{2}} \int d^{d} x \sqrt{g} e^{-2 \varphi}\left(\mathcal{R}+4 \partial^{\mu} \varphi \partial_{\mu} \varphi-\frac{1}{12} H_{\mu \nu \rho} H^{\mu \nu \rho}\right) \\
& -\frac{1}{8}\left(\delta_{i j} F^{i \mu \nu} F_{\mu \nu}^{j}+\delta_{i j} \bar{F}^{i \mu \nu} \bar{F}_{\mu \nu}^{j}-\frac{1}{2} g_{d} \sqrt{\alpha^{\prime}} M_{i j} F_{\mu \nu}^{i} \bar{F}^{j \mu \nu}\right) \\
& -D_{\mu} M_{i j} D_{\nu} M^{i j} g^{\mu \nu}+\frac{16 g_{d}}{\sqrt{\alpha^{\prime}}} \operatorname{det} M+\mathcal{O}\left(M^{4}\right),
\end{aligned}
$$

where the first row contains the universal gravity contribution, the second one contains the gauge field strength for the vector fields of $\mathrm{SU}(2)_{L}$ and $\mathrm{SU}(2)_{R}$ (that we denote here as $A_{L \mu}^{i}, A_{R \mu}^{i}$ respectively). $M_{i j}$ is the matrix of scalars living in the $(\mathbf{3}, \mathbf{3})$ representation. $D_{\mu} M_{i j}=\partial_{\mu} M_{i j}+g_{d} f^{k}{ }_{l i} A_{L \mu}^{l} M_{k j}+g_{d} \bar{f}^{k}{ }_{l j} A_{R \mu}^{l} M_{i k}$ are the usual covariant derivatives and $f_{i j k}=-\bar{f}_{i j k} \propto \epsilon_{i j k}(i, j=1,2,3)$ are the structure constants where $\epsilon_{i j k}$ is the usual Levi-Civita completely antisymmetric tensor and $g_{d}=\kappa_{d} \sqrt{\frac{2}{\alpha^{\prime}}}$.

Interestingly enough, this action can be embedded into an $O(d+3, d+3)$ framework. This is discussed in ref. [3] (and briefly reviewed below) where it was observed that the spectrum of the bosonic string has $(d+3)^{2}$ massless states: $d^{2}$ from $g_{\mu \nu}$ and $B_{\mu \nu}, 6 d$ from the vector states and 9 the scalar states. The number of degrees of freedom precisely agrees with the dimension of the coset

$$
\frac{O(d+3, d+3)}{O(d+3) \times O(d+3)}
$$

that counts the number of degrees of freedom in the DFT formulation with symmetry $O(d+3, d+3)$.

In general a DFT action with $O(D, D)$ symmetry with $D=d+n$, can be written as

$$
\begin{aligned}
& S_{\text {eff }}=\frac{1}{2 \kappa_{d}^{2}} \int d^{d} x \sqrt{g} e^{-2 \varphi}\left[\mathcal{R}+4 \partial^{\mu} \varphi \partial_{\mu} \varphi-\frac{1}{12} H_{\mu \nu \rho} H^{\mu \nu \rho}\right. \\
& -\frac{1}{8} \mathcal{H}_{I J} F^{I \mu \nu} F_{\mu \nu}^{J}+\frac{1}{8}\left(D_{\mu} \mathcal{H}\right)_{I J}\left(D^{\mu} \mathcal{H}\right)^{I J} \\
& -\frac{1}{12} f_{I J}{ }^{K} f_{L M}{ }^{N}\left(\mathcal{H}^{I L} \mathcal{H}^{J M} \mathcal{H}_{K N}-3 \mathcal{H}^{I L} \eta^{J M} \eta_{K N}\right. \\
& \left.\left.+2 \eta^{I L} \eta^{J M} \eta_{K N}\right)-\Lambda\right] .
\end{aligned}
$$

\footnotetext{
${ }^{1}$ Details are presented in next section.
} 
after a generalized Scherk-Schwarz $[5,6]$ like $n$ dimensional compactification. In this expression $\mathcal{H}_{I J}$ with $I, J=1, \ldots, 2 n$ is the, so-called, generalized metric containing the scalar fields coming from the internal components of the $n$-dimensional metric and $B$-field. $\mathcal{R}$ is the $d$-dimensional Ricci scalar and the field strengths $F_{\mu \nu}^{A}$ and $H_{\mu \nu \rho}$ are

$$
\begin{aligned}
F^{I} & =d A^{I}+\frac{1}{\sqrt{2}} f_{J K}{ }^{I} A^{J} \wedge A^{K} \\
H & =d B+F^{I} \wedge A_{I},
\end{aligned}
$$

The covariant derivative of the scalars is

$$
\left(D_{\mu} \mathcal{H}\right)_{I J}=\left(\partial_{\mu} \mathcal{H}\right)_{I J}+\frac{1}{\sqrt{2}} f^{K}{ }_{L I} A_{\mu}^{L} \mathcal{H}_{K J}+\frac{1}{\sqrt{2}} f^{K}{ }_{L J} A_{\mu}^{L} \mathcal{H}_{I K}
$$

The structure constants $f_{N L I}=\eta_{N K} f^{K}{ }_{L I}$ are completely antisymmetric and $\eta_{N K}$ is the $O(n, n)$ metric

$$
\eta^{P Q}=\left(\begin{array}{cc}
1_{n} & 0 \\
0 & -1_{n}
\end{array}\right) .
$$

In our example $D=d+3$, thus $I=1, \ldots 6$. The gauge fields are $A_{\mu}^{I}=\left(A_{L \mu}^{i},-A_{R \mu}^{i}\right)$ and the structure constant splits into

$$
f_{I J}{ }^{K}=\left\{\begin{array}{l}
\left(\frac{2}{\alpha^{\prime}}\right)^{\frac{1}{2}} \epsilon_{i j k} \\
-\left(\frac{2}{\alpha^{\prime}}\right)^{\frac{1}{2}} \bar{\epsilon}_{i j k}
\end{array} .\right.
$$

After expanding around a fixed background the internal generalized metric $\mathcal{H}_{I J}$ can be written as

$$
\mathcal{H} \simeq\left(\begin{array}{cc}
1_{3} & M \\
M^{T} & 1_{3}
\end{array}\right)=I+\left(\begin{array}{cc}
0 & M \\
M^{T} & 0
\end{array}\right)
$$

By replacing above expressions into the action (1.3), and after absorbing constants into the fields, the $\mathrm{SU}(2) \times \mathrm{SU}(2)$ theory given in (1.1) is reproduced. Of course, any reference to DFT could be omitted and just present the above (1.3) action as an interesting way of writing the original expression.

It is worth looking at the term containing the derivatives of scalar fields. Since the metric $\mathcal{H}=I+\ldots$ contains a constant term, the identity, the action could have a contribution,

$$
\begin{aligned}
\left|D_{\mu} \mathcal{H}\right|^{2} & \equiv\left(\cdots+\frac{1}{\sqrt{2}} f^{K}{ }_{L I} A_{\mu}^{L} \delta_{K J}+\frac{1}{\sqrt{2}} f^{K}{ }_{L J} A_{\mu}^{L} \delta_{I K}\right)^{2} \\
& =\cdots+\frac{1}{2}\left(\left[f^{J}{ }_{L I}+f^{I}{ }_{L J}\right] A_{\mu}^{L}\right)^{2} .
\end{aligned}
$$

Namely, a potential "mass term" for the vector bosons.

Moreover, by splitting the $O(n, n)$ indices into Left and Right indices, that we denote as $A=(a, \hat{a})$ and by using that $f_{A B C}=\eta_{A A^{\prime}} f^{A^{\prime}}{ }_{B C}$ is completely antisymmetric, the above term can be recast as

$$
\begin{aligned}
\left(f_{A B C} A_{\mu}^{B} \delta_{D}^{C}+f_{D B C} A_{\mu}^{B} \delta_{A}^{C}\right)^{2} & \sim A_{\mu}^{B} A^{E \mu} f_{A B C} f_{D E F}\left(\eta^{A D} \eta^{C F}-\delta^{A D} \delta^{C F}\right) \\
& \sim A_{\mu}^{B} A^{E \mu} f_{a B \hat{c}} f_{a E \hat{c}}
\end{aligned}
$$


where a sum over repeated indices is understood.

Since in our example $f_{I J K}=\left(f_{i j k}, \bar{f}_{\hat{\hat{j}} \hat{j} \hat{k}}\right)$ the first three "Left" indices do not mix with the last three "Right" ones, such terms vanish.

However, we could envisage a situation where Left and Right indices do mix. In fact, this is what we expect from string theory when we move away from the self dual point. Vertex operators that at the dual point depend only on Left coordinates (or Right coordinates) acquire a mixed dependence and the group breaks down to $\mathrm{U}(1)_{L} \times \mathrm{U}(1)_{R}$ (in the circle example). From this observation we could imagine a description of the symmetry breaking where the structure constants have a dependence on the moduli, namely $f_{I J}{ }^{K}(R)$, such that for the self dual point $R=\tilde{R}=\sqrt{\alpha^{\prime}}$ Left and Right indices do not mix but generically do, away from the fixed point. Let us propose, out of the blue, the following constants

$$
\begin{aligned}
& f_{i j}{ }^{k}=\epsilon_{i j k} \frac{1}{\sqrt{2} \alpha^{\prime}} m_{+}=-f_{\hat{i} \hat{j}}^{\hat{k}} \\
& f_{12}{ }^{\hat{3}}=f_{\hat{1} \hat{2}}{ }^{3}=f_{1 \hat{3}}{ }^{2}=f_{\hat{1} 3}{ }^{\hat{2}}=f_{3 \hat{2}}{ }^{\hat{1}}=f_{\hat{3} 2}{ }^{1}=-\frac{1}{\sqrt{2} \alpha^{\prime}} m_{-}
\end{aligned}
$$

with $\hat{i} \equiv i+3$ and

$$
m_{ \pm}=\frac{1}{R} \pm \frac{1}{\tilde{R}}
$$

If we go back to equation (1.10) and replace above flux values we find

$$
A_{\mu}^{B} A^{E \mu} f_{a B \hat{c}} f_{a E \hat{c}} \propto m_{-}^{2}\left(A_{\mu}^{+}\right)^{2}+m_{-}^{2}\left(A_{\mu}^{-}\right)^{2}
$$

with $A_{\mu}^{ \pm}=A_{\mu}^{1} \pm i A_{\mu}^{2}$ acquiring a mass $m_{-}$whereas $A^{3}$ and $\bar{A}^{3}$ remain massless, indicating that the gauge group is spontaneously broken to $\mathrm{U}(1)_{L} \times \mathrm{U}(1)_{R}$. Moreover, by looking at the couplings $\eta^{I N} \eta^{J M} \partial_{\mu} \mathcal{H}_{N M} f^{K}{ }_{L I} \delta_{K J} A_{\mu}^{L}$ we notice that there is a coupling

$$
\propto m_{-} \partial_{\mu} M_{ \pm, \hat{3}} A^{\mp \mu}
$$

with $M_{ \pm, \hat{3}}=M_{1, \hat{3}}+i M_{2, \hat{3}}$, identifying $M_{ \pm, \hat{3}}$ as would be Goldstone bosons

In fact, by replacing the proposed structure constants in the action (1.3) and after some redefinitions it can be shown that the full string theory effective action [3], computed away from the self-dual point (and keeping slightly massive terms), is reproduced.

Interestingly enough, the structure constants in the action (1.3) can be understood from a DFT perspective. In generalized Scherk-Schwarz reduction of DFT [4-6] they appear as the generalized fluxes of the algebra associated to a generalized vielbein on a doubled internal space. Indeed, it was shown in [3] that such a generalized frame can be explicitly constructed to account for the description of the circle compactification at the self-dual point. In the following sections we indicate how to generalize this frame in order to provide a description valid also (slightly) away from the point of enhancing. The constants presented in (1.11) are then obtained as generalized fluxes from this frame. It is worth emphasizing that, therefore, the resulting DFT construction involves, besides massless states, massive states that become massless at the fixed point. The breaking is not achieved by giving vacuum expectation values (vev's) to scalar fields. We also indicate how to extend the 
construction to toroidal compactifications in more dimensions and provide some examples for the $T^{2}$ case. An interpretation from the string theory point of view is provided as well.

In section 2 we review the basic ideas of Double Field Theory, with special emphasis on the symmetry enhancing situation and by highlighting the ingredients needed in our construction. In particular we discuss how to extend the frame description, away from points of enhancing, from the circle compactification example.

In section 3 we discuss how to extend the DFT construction to describe the enhancingbreaking of gauge symmetries at different points of $m$ dimensional toroidal compactification. The structure of the gauge groups associated to fixed points is known to be of the form $G_{L} \times G_{R}$ where $G_{L}(R)$ are non Abelian gauge groups of rank $m$.

Concluding remarks and a brief outlook are presented in section 4 .

\section{DFT and enhanced gauge symmetries}

Generalized Complex Geometry (GCG) [8-11] and Double Field Theory (DFT) [12-14] are proposals that aim at integrating T-duality as a geometric symmetry. In DFT the presence of windings, an essential ingredient of T-duality, is achieved by introducing new coordinates associated to the winding numbers. Thus in DFT fields depend on a double set of coordinates. This idea, first proposed in [15-20], received new impulse in recent years [21-23] (see [4, 24] for some reviews on the subject and references therein). Generically, these double field theories are constrained theories since some consistency conditions must be satisfied to ensure closure of generalized diffeomorphism algebra. A quite restrictive condition, the so called section condition (or strong constraint), ensures consistency at the price of eliminating half of the coordinates and, therefore, abandoning the original motivation. However, it is worth emphasising that this constrained DFT, which in this case essentially coincides with GCG, still provides an interesting description for understanding underlying symmetries and stringy features (for instance $\alpha^{\prime}$ corrections have been recently incorporated [25-29] in these formulations). An alternative constraint is provided by generalized Scherk-Schwarz like compactifications [30] of DFT [5, 6]. These compactifications contain the generic gaugings of gauged supergravity theories [31, 32] allowing for a geometric interpretation of all of them. In this framework, the double coordinates enter in a very particular way through the twist matrix. Constant gaugings are computed from this matrix and, generically, closure of the algebra is ensure if these gaugings satisfy some quadratic constraints [33] with no need of a strong constraint requirement. A generalization of this formalism was proposed in [3] in order to account for the description of gauge enhancing. The proposal of [3], discussed for the example of circle compactification on $D=d+1$ and inspired in the relation with the coset (1.2), requires to introduce an extended tangent space with $d+1 \rightarrow d+1+2$. However, the "physical space" of DFT is still a double circle. The frame vectors do depend on both circle compact coordinates $y$ and its dual $\tilde{y}$ thus being truly non-geometric. We strongly rely on these results below in order to describe the breaking of enhanced symmetries when moduli do slide slightly away from the fixed points. In this process slightly massive states (those that become massless at the self dual points), with Kaluza Klein(KK) momenta and windings, are expected to appear and are associated 
to an unpaired number $(N \neq \bar{N})$ of Left and Right moving oscillators. We keep these states and disregard other massive states contributions. Such a situation was addressed in [3] from the point of view of string theory. Here we show that DFT is able to capture it. Let us recall that massive states, including winding and momenta, were also considered in DFT context in [34] as generalized Kaluza Klein modes but with equal number of $\mathrm{L}$ and $\mathrm{R}$ oscillators.

In what follows we, briefly, review some basic features of GCG and/or DFT. The theory is defined on a generalized tangent bundle which locally is $T M \oplus T^{*} M$ and whose sections, the generalized vectors $V$, are direct sums of vectors $v$ plus one forms $\xi, V=v+\xi$.

A generalized frame $E_{A}$ on this bundle is a set of linearly independent generalized vectors that belong to the vector space of representations of the group $G=O(D, D)$. It parametrizes the coset $G / G_{c}$, the quotient being over the maximal compact subgroup of $G$. A Lorentz signature is assumed on the $D$-dimensional space-time, i.e. $G_{c}=O(1, D-$ $1) \times O(D-1,1)$. In DFT, this generalized tangent bundle is locally parametrized with a double set of coordinates, $\mathbb{X}^{\mathcal{M}}=\left(x^{\hat{\mu}}, \tilde{x}_{\hat{\mu}},\right)$, defined in the fundamental representation of $O(D, D)$. Here $\mathcal{M}=0, \cdots, 2 D$ and $\hat{\mu}=0, \cdots, D-1$.

A natural pairing between generalized vectors is defined by

$$
V_{1} \cdot V_{2}=\iota_{v_{1}} \xi_{2}+\iota_{v_{2}} \xi_{1}=\eta\left(V_{1}, V_{2}\right)=V_{1}^{\mathcal{M}} \eta_{\mathcal{M N}} V_{2}^{\mathcal{N}}
$$

where the $O(D, D)$ metric $\eta_{\mathcal{M N}}$ has the following off-diagonal form

$$
\eta_{\mathcal{M N}}=\left(\begin{array}{cc}
0 & 1_{D} \\
1_{D} & 0
\end{array}\right)
$$

where $1_{D}$ is the $D \times D$ identity matrix. Note that $\eta_{\mathcal{M N}}$ is invariant under ordinary diffeomorphisms. Defining $\eta_{\mathcal{A B}}=\eta\left(E_{\mathcal{A}}, E_{\mathcal{B}}\right)$ where $\mathcal{A}, \mathcal{B}=0,1, \ldots, 2 D$ are frame indices it results that $\eta_{\mathcal{A B}}$ has the same numerical form as (2.2).

A generalized metric can be constructed as $\mathcal{H}_{\mathcal{M N}}=E^{\mathcal{A}}{ }_{\mathcal{M}} S_{\mathcal{A B}} E^{\mathcal{B}}{ }_{\mathcal{N}}$, where $\mathcal{S}^{\mathcal{A B}}=$ $\operatorname{diag}\left(s^{a b}, s_{a b}\right), s_{a b}$ being the Minkowski metric.

The generalized metric can be parametrized as

$$
\mathcal{H}_{\mathcal{M N}}(\mathbb{X})=\left(\begin{array}{cc}
g^{-1} & -g^{-1} B \\
B g^{-1} & g-B g^{-1} B
\end{array}\right),
$$

satisfying

$$
\mathcal{H}_{\mathcal{M P}} \eta^{\mathcal{P Q}} \mathcal{H}_{\mathcal{Q N}}=\eta_{\mathcal{M N}}
$$

where $g_{\hat{\mu} \hat{\nu}}(\mathbb{X}), B_{\hat{\mu} \hat{\nu}}(\mathbb{X})$ are a symmetric and an anti-symmetric tensor, respectively.

The generalized vectors transform under generalized diffeomorphisms as

$$
\mathcal{L}_{V} W^{\mathcal{M}}=V^{\mathcal{P}} \partial_{\mathcal{P}} W^{\mathcal{M}}+\left(\partial^{\mathcal{M}} V_{\mathcal{P}}-\partial_{\mathcal{P}} V^{\mathcal{M}}\right) W^{\mathcal{P}}
$$

The dilaton field $\varphi$ is incorporated through density field $e^{-2 d}=\sqrt{|g|} e^{-2 \varphi}$ that transforms like a measure

$$
\mathcal{L}_{V} e^{-2 d}=\partial_{P}\left(V^{\mathcal{P}} e^{-2 d}\right)
$$


The algebra of generalized diffeomorphisms closes provided a set of constraints is satisfied. The generalized diffeomorphisms allow to define the generalized dynamical fluxes [4]

$$
\mathcal{F}_{\mathcal{A B C}}=\left(\mathcal{L}_{E_{\mathcal{A}}} E_{\mathcal{B}}\right)^{\mathcal{M}} E_{\mathcal{C M}}
$$

Fluxes are totally antisymmetric in $\mathcal{A B C}$ (flat indices) and transform as scalars under generalized diffeomorphisms, up to the closure constraints.

In generalized Scherk-Schwarz compactifications [4-7] the frame is split into a spacetime piece and an internal one. The former depends on the external $d$-dimensional coordinates $^{2} x^{\mu}$ while the latter strictly depends on the internal $n$-dimensional (where $D=d+n$ ) coordinates $\mathbb{Y}^{I}=\left(\tilde{Y}_{i}, Y^{i}\right)$, defined in the fundamental representation of $O(n, n)$. Here $I=1, \cdots, 2 n$ and

$$
E_{A}(x, Y, \tilde{Y})=\mathcal{U}_{A} A^{\prime}(x) E_{A^{\prime}}^{\prime}(Y, \tilde{Y}) .
$$

The matrix $\mathcal{U}$ encodes the field content in the effective theory, while $E^{\prime}$ is a generalized frame that depends on the internal coordinates. All the dependence on the internal coordinates is through the frame. By using this splitting ansatz the generalized metric becomes $H=\mathcal{S}^{A B} \mathcal{U}_{A}^{A^{\prime}} E_{A^{\prime}}^{\prime} \mathcal{U}_{B}^{B^{\prime}} E_{B^{\prime}}^{\prime}=\mathcal{H}^{A^{\prime} B^{\prime}} E_{A^{\prime}}^{\prime} E_{B^{\prime}}^{\prime}$ where all the field dependence on space time coordinates is encoded in

$$
\mathcal{H}^{A^{\prime} B^{\prime}}(x)=\mathcal{S}^{A B} \mathcal{U}_{A} A^{\prime} \mathcal{U}_{B}^{B^{\prime}}
$$

parametrizing the moduli space. In particular, we will deal with the "internal piece" $\mathcal{H}^{I J}$, where $I, J=1, \ldots, 2 n$ are frame indices on the internal part of the double tangent space.

It proves useful to rotate to a Right-Left basis $\mathcal{C}$ where left and right coordinates are

$$
\begin{aligned}
y_{L m} & =\frac{1}{2}\left[(g+B)_{m n} y^{n}+\tilde{y}_{m}\right] \\
y_{R m} & =\frac{1}{2}\left[(g-B)_{m n} y^{n}-\tilde{y}_{m}\right]
\end{aligned}
$$

in terms of $\mathbb{Y}_{M}=\left(y_{m}, \tilde{y}^{m}\right)$. Namely, the rotation matrix reads

$$
R=\left(\begin{array}{cc}
(g+B) & 1 \\
(g-B) & -1
\end{array}\right)
$$

and therefore from $E_{A} \rightarrow\left(E_{\mathcal{C}}\right)_{A}=R_{A}^{B} E_{B}$ we see that $\eta$ becomes diagonal

$$
\left(R \eta R^{T}\right)_{A B}=\left(\begin{array}{cc}
1_{D} & 0 \\
0 & -1_{D}
\end{array}\right) .
$$

Since the internal piece of $H$ lies in $O(n, n) / O(n) \times O(n)$ it is possible to show [3, 44] that the scalar matrix, in the Left-Right basis $\mathcal{C}$ can be written as an expansion in scalar fluctuations

$$
\mathcal{H}_{\mathcal{C}}=\left(\begin{array}{cc}
1_{n}+M M^{T} & M \\
M^{T} & 1_{n}+M^{T} M
\end{array}\right)+O\left(M^{3}\right)
$$

with $n^{2}$ independent degrees of freedom.

\footnotetext{
${ }^{2}$ The $\tilde{x}^{\mu}$ duals are dropped off, or equivalently the strong constraint is imposed in the space time sector.
} 
By using the expression for the generalized Lie derivative in the specific case of the frame

$$
\begin{aligned}
\mathcal{L}_{E_{A}^{\prime}} E_{B}^{\prime} & =\frac{1}{2}\left[E_{A}^{\prime P} \partial_{P} E_{B}^{\prime M}-E_{B}^{\prime P} \partial_{P} E_{A}^{\prime M}+\eta^{M N} \eta_{P Q} \partial_{N} E_{A}^{\prime P} E_{B}^{\prime Q}\right] D_{M} \\
{\left[E_{I}^{\prime}, E_{J}^{\prime}\right] } & =\mathcal{L}_{E_{I}^{\prime}} E_{J}^{\prime}=f_{I J}{ }^{K} E_{K}^{\prime} .
\end{aligned}
$$

where the fluxes $f_{I J}{ }^{K}$, for the generalised Scherk-Schwarz reduction, must be constants and must satisfy the constraints

$$
f_{I J K} \equiv \eta_{K L} f_{I J}^{L}=f_{[I J K]}, \quad f_{[I J}^{L} f_{K] L}^{R}=0 .
$$

The information about the internal space is encoded in these constants. When replacing above results into the initial DFT action (1.3) the expression presented in (1.1) is obtained.

\subsection{Enhanced gauge symmetry on the circle}

In ref. [3] a specific DFT frame was presented ${ }^{3}$ in order to reproduce the effective action, obtained from string theory compactification on the circle, at the self-dual point. As mentioned it requires to enhance the tangent space to $D=d+1+2$ but the frame only depends on the circle coordinate and its dual. In a Cartan-Weyl basis the frame vectors read,

$$
\begin{array}{lll}
E_{ \pm}=c\left(e^{\mp i \frac{2}{\sqrt{\alpha^{\prime}}} y_{L}}, i e^{\mp i \frac{2}{\sqrt{\alpha^{\prime}}} y_{L}}, 0,0,0,0\right), & & E_{3}=-c(0,0,1,0,0,0,0) \\
\bar{E}_{\hat{1}}=c\left(0,0,0, e^{\mp i \frac{2}{\sqrt{\alpha^{\prime}}} y_{R}}, i e^{\mp i \frac{2}{\sqrt{\alpha^{\prime}}} y_{R}}, 0\right) & \bar{E}_{\hat{3}}=-c(0,0,0,0,0,1)
\end{array}
$$

The directions $E_{\hat{1}} \equiv E_{1}+i E_{2}$ (and $\bar{E}_{\hat{1}}=E_{\hat{1}}+i E_{\hat{2}}$ ) encode the extension of the tangent space. It is easy to check that, by using (2.14) (setting $c=i \sqrt{\alpha^{\prime}}$ ) and by noticing that the only contributions to the partial derivative are

$$
\partial_{A}=\left(0,0, \partial_{y_{L}}, 0,0, \partial_{y_{R}}\right),
$$

the $\mathrm{SU}(2)_{L} \times \mathrm{SU}(2)_{R}$ coupling constants (1.7) are obtained. In the Cartan-Weyl basis they read,

$$
\begin{aligned}
& \frac{1}{2} f_{+-}{ }^{3}=\frac{1}{2} f_{\hat{\hat{+}} \hat{-}^{\hat{3}}}=f_{3+}{ }^{+}=f_{\hat{3} \hat{+}}{ }^{\hat{+}}=-f_{3-}{ }^{-}=-f_{\hat{3} \dot{\bar{\alpha}}} \hat{\hat{-}}=1 \\
& -\frac{1}{2} f_{\hat{+} \hat{-}}{ }^{3}=-\frac{1}{2} f_{+-}{ }^{\hat{3}}=f_{3 \hat{+}}{ }^{\hat{+}}=-f_{3 \hat{\mathcal{C}}^{\hat{-}}}=f_{\hat{3}+}+=-f_{\hat{3}-}{ }^{-}=0
\end{aligned}
$$

where we have used a hat to denote the indices constructed up from 4, 5, 6 Right indices.

The construction of the frame is inspired in the coset structure (1.2) and on the structure of vertex operators in string theory. ${ }^{4}$ Namely the correspondence among frame vectors and string current generators [3] can be established (here $e^{ \pm L}=e_{1} \pm i e_{2}, \quad e^{ \pm R}=e^{1} \pm i e^{2}$ )

$$
\begin{aligned}
\bar{E}^{ \pm}=e^{\mp \frac{2 i}{\sqrt{\alpha^{\prime}}} y^{R}} e^{ \pm R} & \leftrightarrow e^{\mp \frac{2 i}{\sqrt{\alpha^{\prime}}} y^{R}(\bar{z})} d \bar{z}=\bar{J}^{\mp} d \bar{z}, \\
\bar{E}^{3}=i / \sqrt{\alpha^{\prime}} d y^{R} & \leftrightarrow d y^{R}(\bar{z})=\bar{J}^{3} d \bar{z} \\
E^{ \pm}=e^{\mp \frac{2 i}{\sqrt{\alpha^{\prime}}} y^{L}} e^{ \pm L} & \leftrightarrow e^{\mp \frac{2 i}{\sqrt{\alpha^{\prime}}} y^{L}(z)} d z=J^{\mp} d z, \\
E^{3}=i / \sqrt{\alpha^{\prime}} d y^{L} & \leftrightarrow d y^{L}(z)=J^{3} d z .
\end{aligned}
$$

\footnotetext{
${ }^{3}$ The choice of frame was inspired by a previous work [35] set in a different context.

${ }^{4}$ Basic ingredients and notation conventions for string theory vertices are briefly presented in appendix.
} 
where $J^{\mp}(z)=e^{\mp \frac{2 i}{\sqrt{\alpha^{\prime}}} y^{L}(z)}, J^{3}(z)=\partial_{z} Y(z)$ are the string currents satisfying the Operator Product Expansion (OPE) algebra of $\mathrm{SU}(2)_{L}$ (and similarly for the Right sector). The corresponding string vertex operators $V^{i}(z, \bar{z})$ for vectors are

$$
V^{ \pm, 3}(z, \bar{z})=i \frac{g_{c}^{\prime}}{\alpha^{\prime 1 / 2}} \epsilon_{\mu}^{ \pm, 3}: J^{ \pm, 3}(z) \bar{\partial} X^{\mu} e^{i K \cdot X}:
$$

where $i= \pm, 3$ and $K^{\mu}$ is the space time momentum.

A similar construction was presented in [36] (see also [37]) for the case of the $S^{3}$ reduction in the context of the WZW model, inspired by [38]. The purely geometric case was studied in [39]. For the non-geometric one [36], the authors were able to show that allowing for a non trivial dependence on the dual coordinate of the Hopf fibre, non-geometric gaugings can be obtained [40]. However, unlike the toroidal construction presented here (and in [3]) where a clear world sheet picture arises, the $S^{3}$ does not have non-contractible cycle and, therefore, no winding states were really considered in [36].

For general compactification radios, the dependence on moduli is encoded in the exponential part of the vertex operators

$$
: \exp \left[i k_{L} y_{L}(z)+i k_{R} y_{R}(\bar{z})\right] e^{i K \cdot X}:
$$

where

$$
k_{L}^{(p, \tilde{p})}=\frac{p}{R}+\frac{\tilde{p}}{\tilde{R}}, \quad k_{R}^{(p, \tilde{p})}=\frac{p}{R}-\frac{\tilde{p}}{\tilde{R}} .
$$

in terms of KK momenta $p$ and winding number $\tilde{p}$ satisfying the level matching condition $\bar{N}-N=p \tilde{p} . \quad N=N_{x}+N_{y}\left(\bar{N}=\bar{N}_{x}+\bar{N}_{y}\right)$ is the Left (Right) moving number operator, involving the sum of the number operator along the circle $N_{y}\left(\bar{N}_{y}\right)$ and the number operator for the non-compact space-time directions, denoted by $N_{x}\left(\bar{N}_{x}\right)$. At the self-dual radio $R=$ $\tilde{R}=\sqrt{\alpha}^{\prime}$, the vertices separate into a Left part with $k_{R}=0$ or into a Right vertices with $k_{L}=0$. The three vector states generating $\mathrm{SU}(2)_{L}$ correspond to $\bar{N}_{x}=1, N_{x}=\bar{N}_{y}=0$. The assignment $N_{y}=1, p=\tilde{p}=0$ corresponds to the KK (Cartan field) mode $A_{L \mu}^{3}$, while for $N_{y}=0, p=\tilde{p}= \pm 1$ (namely, $\left.k_{L}^{( \pm 1, \pm 1)}= \pm \frac{2}{\sqrt{\alpha^{\prime}}} ; k_{R}^{( \pm 1, \pm 1)}=0\right)$ the charged vectors $A_{L \mu}^{ \pm}$ are obtained (and similarly for $\mathrm{SU}(2)_{R}$ ).

When moving away from the fixed point, Left and Right parts mix up and, generically, the original vertex operator becomes ill defined as a conformal field. It must combine with other vertex operators, that have the same exponential contribution, in order to produce a new consistent vertex. Interestingly enough, these combinations encode the Higgs mechanism by absorption of a vertex corresponding to a would be Goldstone boson field [3]. With this picture in mind we generalize the frame (2.17) by including the dependence $k_{L} y_{L}+k_{R} y_{R}$ for the found values of momenta and windings.

$$
\begin{aligned}
E_{ \pm} & =c\left(e^{\mp i w}, \pm i e^{\mp i w}, 0,0,0,0\right) & \bar{E}_{+} & =c\left(0,0,0, e^{\mp i \bar{w}}, \pm i e^{i \mp \bar{w}}, 0\right) \\
E_{3} & =-c(0,0,1,0,0,0,0) & \bar{E}_{\hat{3}} & =-c(0,0,0,0,0,1)
\end{aligned}
$$

where

$$
w=m_{+} y_{L}+m_{-} y_{R}, \quad \bar{w}=m_{-} y_{L}+m_{+} y_{R}
$$


and $m_{ \pm}=k_{L}^{(1, \pm 1)}=\frac{1}{R} \pm \frac{1}{\tilde{R}}$. Notice that $m_{+} \rightarrow \frac{2}{\sqrt{\alpha^{\prime}}}$ at self-dual radio $R=\tilde{R}=\sqrt{\alpha^{\prime}}$. Again, by using (2.14) we obtain

$$
\begin{aligned}
& {\left[E_{+}, E_{-}\right]=2\left(a_{+} E_{3}+a_{-} \bar{E}_{\hat{3}}\right), \quad\left[E_{\hat{+}}, \bar{E}_{\hat{-}}\right]=2\left(a_{+} \bar{E}_{\hat{3}}-a_{-} E_{3}\right),} \\
& {\left[E_{3}, E_{+}\right]=a_{+} E_{+}, \quad\left[\bar{E}_{\hat{3}}, \bar{E}_{\hat{+}}\right]=a_{+} \bar{E}_{\hat{+}}} \\
& {\left[E_{3}, E_{-}\right]=-a_{+} E_{-}, \quad\left[E_{\hat{3}}, \bar{E}_{\hat{\imath}}\right]=-a_{+} \bar{E}_{\hat{\imath}}} \\
& {\left[\bar{E}_{\hat{3}}, E_{+}\right]=a_{-} E_{+}, \quad\left[E_{3}, \bar{E}_{\hat{+}}\right]=a_{-} E_{\hat{+}}} \\
& {\left[\bar{E}_{\hat{3}}, E_{-}\right]=-a_{-} E_{-}, \quad\left[E_{3}, \bar{E}_{\hat{\iota}}\right]=-a_{-} \bar{E}_{\hat{\iota}}}
\end{aligned}
$$

with

$$
a_{ \pm}=\frac{\sqrt{\alpha^{\prime}} m_{ \pm}}{2}
$$

Thus, we find that, by computing the fluxes (2.16), and up to a normalization factor $\alpha^{\prime \frac{3}{2}} \sqrt{2}$, the constants proposed in (1.11) are obtained (here written in a complex combination). Notice that, if $R \rightarrow \tilde{R}$ then $a_{-}\left(a_{+}\right) \rightarrow 0(1)$ and the original $\mathrm{SU}(2) \times \mathrm{SU}(2)$ algebra is recovered. Moreover, it is easy to check that the algebra is invariant under T-duality transformation $R \leftrightarrow \tilde{R}$.

As mentioned, by systematically replacing the above structure constants (fluxes) into the general DFT action expression (1.3), the exact spontaneously broken action, with $\mathrm{U}(1) \times \mathrm{U}(1)$ gauge symmetry, as computed from string theory (see eq. (3.31) in [3]) is found. In particular, vector fields $A_{L \mu}^{ \pm}$and $A_{R \mu}^{\hat{ \pm}}$ become massive, with masses $m_{-}$by "eating" the would be Goldstone bosons $\partial_{\mu} M_{ \pm \hat{3}}$ (and $\partial_{\mu} M_{3 \hat{x}}$ ) that disappear from the spectrum.

It appears instructive to see how some of the terms in the broken symmetry action arise. For instance, by inserting the expansion in scalar fluctuations $M$ in the generalized scalar matrix (2.13), into the third row of the DFT action (1.3) and using the values (1.11) for structure constants we find the quadratic terms

$$
\begin{aligned}
2\left(m_{+} m_{-}\right. & \left.+m_{-}^{2}\right)\left|M^{ \pm \pm}\right|^{2}-2\left(m_{+} m_{-}-m_{-}^{2}\right)\left|M^{ \pm \mp}\right|^{2} \\
& =\frac{4}{R} m_{-}\left|M^{ \pm \pm}\right|^{2}-\frac{4}{R} m_{-}\left|M^{ \pm \mp}\right|^{2}
\end{aligned}
$$

reproducing the exact values $m_{ \pm \pm}^{2}=\frac{4}{R} m_{-}$and $m_{ \pm \mp}^{2}=-\frac{4}{R} m_{-}$as computed from string mass formula (A.2). ${ }^{5}$ The terms proportional to $m_{+} m_{-}$and $m_{-}^{2}$ come from linear and quadratic terms in $M$ expansion in (2.13), respectively.

In the same way it can be checked that the masses of the would be Goldstone bosons $M^{ \pm, \hat{3}}, M^{3 \hat{ \pm}}$ coincide, as it should, with the masses $m_{-}$of the massive vector bosons.

Moreover, the same row in (1.3) for cubic terms in $M$ lead to

$$
-\frac{4}{\sqrt{\alpha^{\prime}}} M_{+-} M_{-+} M_{33}\left(\frac{\sqrt{\alpha^{\prime}}}{\tilde{R}}\right)^{2}+\frac{4}{\sqrt{\alpha^{\prime}}} M_{++} M_{--} M_{33}\left(\frac{\sqrt{\alpha^{\prime}}}{R}\right)^{2}
$$

with $m_{+}^{2}+m_{-}^{2}+2 m_{+} m_{-}=\left(m_{+}+m_{-}\right)^{2}=\frac{4}{R^{2}}$.

\footnotetext{
${ }^{5}$ Recall that, depending on the value of $R$ half of the scalars become tachyonic. This is an artefact associated to the ill defined bosonic string.
} 
Coming back to the expressions (2.27), it is worth noticing that the above brackets close into a Lie algebra for arbitrary values of $R$. Indeed, by recalling that $f_{I J K}=\eta_{K L} f_{I J}{ }^{L}$ are totally antisymmetric, it is easy to check that Jacobi identity is satisfied. Of course, the found algebra should correspond to one of the known semi-simple algebras. Since it involves six charged generators and two Cartan ones the only possibility is $\mathrm{SU}(2) \times \mathrm{SU}(2)$. Actually, this can be explicitly shown by performing the linear combinations of generators

$$
\begin{aligned}
E_{ \pm}^{\prime} & =E_{ \pm} ; \quad \bar{E}_{\hat{x}}^{\prime}=\bar{E}_{\hat{1}} \\
E_{3}^{\prime} & =a_{+} E_{3}-a_{-} \bar{E}_{\hat{3}} \\
E_{\hat{3}}^{\prime} & =-a_{-} E_{3}+a_{+} \bar{E}_{\hat{3}},
\end{aligned}
$$

namely a rotation by the $O(3,3)$ matrix

$$
\left(\begin{array}{cccc}
1_{2} & 0 & 0 & 0 \\
0 & a_{+} & 0 & -a_{-} \\
0 & 0 & 1_{2} & 0 \\
0 & -a_{-} & 0 & a_{+}
\end{array}\right)
$$

and using that $a_{+}^{2}-a_{-}^{2}=1$.

We thus see that, even in the broken phase, there is still an underlying $\mathrm{SU}(2)$ symmetry (now mixing massive and massless states). However, once the above frame is chosen, the $O(3,3)$ full symmetry gets broken and, therefore, it can not be rotated to the starting point. Recall also that, in terms of fields, the combination of $\mathrm{U}(1)$ gauge bosons

$$
A^{3^{\prime} \mu}=a_{-} A_{L}^{3 \mu}+a_{+} A_{R}^{\hat{3} \mu}=V^{\mu}+B^{\mu}
$$

is the right combination in terms of

$$
V_{\mu}=\frac{1}{2 R}\left(A_{\mu}^{3}+\bar{A}_{\mu}^{3}\right), \quad B_{\mu}=\frac{1}{2 \tilde{R}}\left(A_{\mu}^{3}-\bar{A}_{\mu}^{3}\right)
$$

which are the KK reductions of the metric and antisymmetric fields and with respect to which massive states carry integer charge (see [3]).

It is instructive to look at the above results from the string theory point of view. There, the structure constants can be essentially read from the 3-gauge vector bosons vertices with vertex operators $V^{i}$. For the massless case they read (see [3] for notations and explicit computations), for Left vectors,

$$
\begin{gathered}
\left\langle V_{L}^{i} V_{L}^{j} V_{L}^{k}\right\rangle=\pi g_{c} \frac{i}{\sqrt{\alpha^{\prime}}} \epsilon^{i j k}\left[\left(\epsilon_{3}^{k} \cdot K_{1}\right)\left(\epsilon_{1}^{i} \cdot \epsilon_{2}^{j}\right)-\left(\epsilon_{2}^{j} \cdot K_{1}\right)\left(\epsilon_{1}^{i} \cdot \epsilon_{3}^{k}\right)\right. \\
\left.+\left(\epsilon_{1}^{i} \cdot K_{2}\right)\left(\epsilon_{3}^{k} \cdot \epsilon_{2}^{j}\right)\right]
\end{gathered}
$$

where $K_{1}, K_{2}, K_{3}$ are the space time momenta of vertices $i, j, k$ respectively. Namely, we can read the $\epsilon^{i j k}$ structure constants of $\mathrm{SU}(2)_{L}$ (and similarly for $\mathrm{SU}(2)_{R}$ ) and there is no mixing between L-R sectors. 
On the other hand, away from the self-dual point we find the three-point coupling of Left and Right vectors can be written as

$$
\begin{aligned}
\left\langle V_{L}^{+} V_{L}^{-} V_{L}^{3}\right\rangle & =\frac{\pi g_{c}^{\prime}}{2 \sqrt{\alpha^{\prime}}}\left(a_{+}\right) E\left(K_{i}, \epsilon_{i}\right) \\
\left\langle V_{L}^{+} V_{L}^{-} V_{R}^{3}\right\rangle & =\frac{\pi g_{c}^{\prime}}{2 \sqrt{\alpha^{\prime}}}\left(a_{-}\right)\left(\epsilon_{1+}^{\prime} \cdot \epsilon_{2-}^{\prime}\right)\left(K_{1} \cdot \epsilon_{3}\right) \\
\left\langle V_{R}^{+} V_{R}^{-} V_{L}^{3}\right\rangle & =\frac{\pi g_{c}^{\prime}}{2 \sqrt{\alpha^{\prime}}}\left(a_{-}\right)\left(\epsilon_{1+}^{\prime} \cdot \epsilon_{2-}^{\prime}\right)\left(K_{1} \cdot \epsilon_{3}\right)
\end{aligned}
$$

where $E\left(K_{i}, \epsilon_{i}\right)=\left(\epsilon_{1+}^{\prime} \cdot \epsilon_{2-}^{\prime}\right)\left(K_{1} \cdot \epsilon_{3}\right)+\left(\epsilon_{1+}^{\prime} \cdot \epsilon_{3}\right)\left(K_{3} \cdot \epsilon_{2}^{\prime}\right)+\left(\epsilon_{3} \cdot \epsilon_{2-}^{\prime}\right)\left(K_{2} \cdot \epsilon_{1+}^{\prime}\right)$ is a factor that depends on space time momenta and vector polarizations. Thus, if by analogy with the dual point case, we interpret the coefficients as the moduli dependent coupling constants we find; $f_{+-3}(R)=a_{+}, f_{+-\hat{3}}(R)=a_{-}$etc. Namely, we are extracting the structure constants $f^{A B C}$, away from the self dual point, from the three current amplitudes $\left\langle J^{A} J^{B} J^{C}\right\rangle$. Moreover, by considering the combinations (2.33) above, we can again identify the underlying $\mathrm{SU}(2)$ structure. The SU(2) controls the allowed three point functions through conservation of internal Right and Left momenta.

\section{Enhancing-breaking of gauge symmetries for generic toroidal compact- ifications}

In this section we briefly discuss possible realizations of the enhanced symmetry breaking mechanism, through moduli dependent structure constants, for general toroidal compactifications. Bosonic string compactification [2] on a $T^{r}$ torus of $r$ dimensions gives rise to a gauge symmetry group $G_{L} \times G_{R}$ of rank $2 r$ ( $r$ coming from Left and Right vectors associated to the metric and $B$ field degrees of freedom). At generic points of the compactified manifold this group is simply $\mathrm{U}(1)_{L}^{r} \times \mathrm{U}(1)_{R}^{r}$ but, at special moduli points, $G_{L}$ is a non abelian group with $\operatorname{dim} G_{L}=n=n_{c}+r$. Here $n_{c}$ counts the number of charged generators associated to the presence of non trivial winding and KK momenta. By reasoning as in the circle case, if we assume that the number of massless degrees of freedom at some point of enhancing is given by

$$
\operatorname{dim}\left(\frac{O(d+n, d+n)}{O(d+n) \times O(d+n)}\right)=d^{2}+2 n d+n^{2}
$$

this appears to correspond to the $d^{2}$ degrees of freedom of gravity (plus B field), the $2 n$ vectors of a $G_{L} \times G_{R}$ and $n^{2}$ scalars in bi-adjoint representations. If $n=r$ it gives the correct counting for $\mathrm{U}(1)_{L}^{r} \times \mathrm{U}(1)_{R}^{r}$ degrees of freedom.

For a circle compactification $r=1$ and by choosing $n=2+1$ the counting corresponds to an $\mathrm{SU}(2)_{L} \times \mathrm{SU}(2)_{R}$ gauge group with scalars in the $(\mathbf{3}, \mathbf{3})$ representation, as it is the case for the self-dual point $R=\tilde{R}$.

For a $T^{2}$ toroidal compactification, when $n=2 \times 3$, the number of massless degrees of freedom for $\mathrm{SU}(2)_{L} \times \mathrm{SU}(2)_{L} \times \mathrm{SU}(2)_{R} \times \mathrm{SU}(2)_{R}$ with scalars in $(\mathbf{3}, \mathbf{1}, \mathbf{3}, \mathbf{1})+(\mathbf{1}, \mathbf{3}, \mathbf{1}, \mathbf{3})+$ $(\mathbf{3}, \mathbf{1}, \mathbf{1}, \mathbf{3})+(\mathbf{1}, \mathbf{3}, \mathbf{3}, \mathbf{1})$, corresponding to a possible torus enhancing point, is reproduced. 
Also, the correct counting occurs for $n_{c}=6$, for the degrees of freedom of $\mathrm{SU}(3)_{L} \times \mathrm{SU}(3)_{R}$ with scalars in the $(\mathbf{8}, \mathbf{8})$ representation at the point of maximal enhancing [2].

The generalization of the exponential contribution (2.23) to the string vertex operators for a general torus (with lattice vectors $e^{a}{ }_{m}$ ) reads (see appendix for notation)

$$
: e^{i k_{L} \cdot y_{L}(z)+i k_{R} \cdot y_{R}(\bar{z})} e^{i K \cdot X}:
$$

with Left and Right momenta

$$
k_{L}^{a}=e^{a}{ }_{m} p_{L}^{m}, \quad k_{R}^{a}=e^{a}{ }_{m} p_{R}^{m}
$$

where

$$
p_{L}^{m}=\tilde{p}^{m}+g^{m n}\left(p_{n}-B_{n k} \tilde{p}^{k}\right), \quad p_{R}^{m}=-\tilde{p}^{m}+g^{m n}\left(p_{n}-B_{n k} \tilde{p}^{k}\right) .
$$

$g_{m n}=e^{a}{ }_{m} e^{a}$ defines the internal metric whereas $B_{m n}$ are the internal components of the Kalb-Ramond field.

Notice that, by using (2.11) the following relation holds

$$
k_{L} \cdot y_{L}+k_{R} \cdot y_{R}=\mathbb{P} \cdot \mathbb{Y}
$$

Gauge symmetry enhancing occurs at specific values of moduli $\left(g_{0}, B_{0}\right)$, encoded in the frame vectors $e_{m}^{a}(g, B)$ and of windings and momenta (encoded in the generalized momentum $\mathbb{P}=\left(p_{1}, p_{2}, \ldots ; \tilde{p}^{1}, \tilde{p}^{2} \ldots\right)$. At such values, $k_{L}^{a}$ become roots of a semi simple algebra $\left(k_{R}^{a}=0\right)$ and similarly for the right sector. Namely, at such points, the internal part of vertex operators in (3.2) becomes

$$
E_{\alpha} \simeq e^{i k_{L}^{\mathbb{P}} \cdot y(z)}
$$

with $k_{L}^{(\mathbb{P}) a} \equiv \alpha_{m}^{a}$ a root of the semi simple algebra and where $m=1,2, \ldots$ (associated to $\mathbb{P}$ values) labels the charged operators. These vertex operators, together with the corresponding Cartan operators, close the OPE of a $G_{L}$ group affine algebra.

Let us consider the 2-torus example discussed in the appendix. For generic values of $E=g+B$ the gauge group is $\mathrm{U}(1)_{L}^{2} \times \mathrm{U}(1)_{R}^{2}$ but enhancings occur at different points [2]. For instance, by choosing the basis ${ }^{6} e_{m}=\frac{1}{\sqrt{2}} \alpha_{m}$ with $m=1,2$ with $\alpha_{1,2}$ the simple roots of $\mathrm{SU}(3)$ and $B_{12}=g_{12}=-\frac{1}{2}$ we see that there are six generalized momentum vectors

$$
\mathbb{P}= \pm(1,0,1,0), \pm(-1,1,0,1), \pm(0,1,1,1)
$$

that satisfy the LMC, and such that $P_{R}^{m}=0$. They give rise to six extra massless states with

$$
P_{L}= \pm(1,0), \pm(0,1), \pm(1,1)
$$

Similarly $\mathbb{P}= \pm(-1,1,1,0), \pm(0,-1,0,1), \pm(1,0,-1,-1)$ lead to same roots for $P_{R}$ while $P_{L}=0$. At the end, the enhanced $\mathrm{SU}(3)_{L} \times \mathrm{SU}(3)_{R}$ gauge group is generated.

\footnotetext{
${ }^{6}$ The $\sqrt{2}$ is just a normalization factor in order to keep the usual convention for $\alpha^{2}=2$ for the roots.
} 
Also, for

$$
G_{m n}=\left(\begin{array}{ll}
1 & 0 \\
0 & 1
\end{array}\right)
$$

and $B=0$ an enhancing to $(\mathrm{SU}(2) \times \mathrm{SU}(2))_{L} \times(\mathrm{SU}(2) \times \mathrm{SU}(2))_{R}$ is obtained for $\mathbb{P}=$ $( \pm 1,0, \pm 1,0),(0, \pm 1,0, \pm 1)$, etc.

The description of the enhancing-breaking of the gauge symmetry could be, in principle, described by generalizing the steps presented in the previous section for the circle particular situation. We have not pursued this construction systematically but we present some examples for the 2-torus case. ${ }^{7}$ For a general $r$-torus compactification, from a DFT point of view, we should consider a doubling of the internal manifold $T^{r} \times \tilde{T}^{r}$, incorporating both tori coordinates $y^{m}$ as well as their duals $\tilde{y}_{m}$ with $m=1, \ldots r$ ( in a $O(r, r)$ writing it corresponds to the double coordinate $\mathbb{Y}^{M}$ with $M=1, \ldots 2 r$.).

Following the counting (3.1) it appears that the tangent frame must be enlarged further in order to incorporate information about charged operators. Thus, if we were to describe a $G_{L} \times G_{R}$ point, besides the $r+r$ frame vectors, associated to the internal coordinates Cartan generators, $2 n_{c}$ extra frame vectors should be incorporated with $n_{c}=(\operatorname{dim} G-r)$ associated to the left charged generator vertices (3.5) (and similarly for Right vertices). Thus, in principle, we should have a $2 \operatorname{dim} G$ tangent frame space where the frame vectors only depend on $\mathbb{Y}$ internal coordinates. Each frame vector could be written in a given $2 \operatorname{dim} G$ basis and frame vectors associated to charged operators are expected to depend on an exponential factor $e^{i k_{L}^{(\mathbb{P})} \cdot y_{L}}$ (and similarly for $\mathrm{R}$ vectors) where $\mathbb{P}$ encodes the specific values of momenta and winding characterizing the enhanced vectors.

For generic points in the compact manifold we will have internal directions $e_{m}^{a}(g, B)$ depending on the moduli fields and, therefore, so do $k_{L}^{(\mathbb{P})}(g, B)$ and $k_{R}^{(\mathbb{P})}(g, B){ }^{8}$ At selected values of $g, B$ these directions become the simple roots of the enhancing algebra. Therefore, away from fixed points we expect the frame vectors to depend on both $e^{i k_{L}^{(\mathbb{P})} \cdot y_{L}+i k_{R}^{(\mathbb{P})} \cdot y_{R}}$, as in fact, we found in the circle case (recall that the possible values of $\mathbb{P}$ are fixed). When moving into the fixed point, $\mathbb{P}$ values will produce the roots of $G_{L}$ (and $k_{R}^{\mathbb{P}}=0$ ) and the roots of $G_{R}$ (and $k_{L}^{\mathbb{P}}=0$ ). This is indeed what we found in the circle case and we now illustrate in its simplest generalization of the 2-torus case near the $\mathrm{SU}(2)^{4}$ fixed point.

Let us name $\mathbb{Y}^{M}=\left(\tilde{y}_{m}, y^{m}\right), m=1,2$, the double torus coordinates or $\left(y_{L m}, y_{R m}\right)$ in a $L-R$ basis. The exponential contributions can now be written in terms of $e^{i \theta_{j}}$ where

$$
\theta_{(j)}=k_{(j) L}^{m} y_{L m}+k_{(j) R}^{m} y_{R m}=k_{(j) L}^{1} y_{L 1}+k_{(j) L}^{2} y_{L 2}+k_{(j) R}^{1} y_{R 1}+k_{(j) L}^{2} y_{R 2}
$$

Here $(j)$ encodes the $\mathbb{P}=\left(p_{1}, p_{2}, \tilde{p}^{1}, \tilde{p}^{2}\right)$ values that would lead to $\mathrm{SU}(2)_{j}$ at the self-dual point. For instance, $\mathbb{P}=( \pm 1,0, \pm 1,0)$ generates a $k_{(1) L}^{m}$ and $k_{(1) R}^{m}\left(\right.$ where $k_{(1) R}^{m}=0$ at

\footnotetext{
${ }^{7}$ A systematic derivation is proposed in [44] with a modification of the generalized Lie derivative.

${ }^{8}$ We avoid writing the dependence on moduli in order to lighten the notation.
} 
self-dual point) etc. Overall we find

$$
\begin{aligned}
& \mathbb{P}=( \pm 1,0, \pm 1,0) \rightarrow k_{(1) L(R)}^{m} \\
& \mathbb{P}=(0, \pm 1,0, \pm 1) \rightarrow k_{(2) L(R)}^{m} \\
& \mathbb{P}=( \pm 1,0, \mp 1,0) \rightarrow k_{(3) L(R)}^{m} \\
& \mathbb{P}=(0, \pm 1,0, \mp 1) \rightarrow k_{(4) L(R)}^{m}
\end{aligned}
$$

where at the corresponding self-dual point $k_{(1) R}^{m}=k_{(2) R}^{m}=0$ and $k_{(3) L}^{m}=k_{(4) L}^{m}=0$. Following the general steps sketched above we thus propose a generalized twelve dimensional ( $2 \operatorname{dim} G_{L}=12$ ) frame with frame vectors depending only on $\mathbb{Y}^{M}$. A straightforward generalization of the circle case leads us to the frame vectors

$$
\begin{aligned}
E_{+(j)} & =\left(0^{3(j-1)} ; \boldsymbol{v}_{+(j)} ; 0^{3(4-j)}\right) e^{-i \theta_{j}}=E_{-(j)}^{*} \\
E_{0(j)} & =\left(0^{3(j-1)} ; \boldsymbol{v}_{0(j)} ; 0^{3(4-j)}\right)
\end{aligned}
$$

where $\boldsymbol{v}_{ \pm j}=(0,1, \pm i)\left(\boldsymbol{v}_{0 j}=(i, 0,0)\right)$ is a 3 dim vector inserted at position $j$. Notice that $E_{+(j+3)} \equiv \bar{E}_{+(j)}$ correspond to Right vectors. At the self-dual point these vectors lead to $\mathrm{SU}(2)_{L} \times \mathrm{SU}(2)_{R}$ algebra for each value of $j$.

Moving away from the $\mathrm{SU}(2)^{4}$ fixed point generically mix the twelve generators leading to moduli dependent structure constants $f_{I J K}(g, B)(I, J, K=1, \ldots 12)$. Actually, due to the frame structure (3.12), the mixing occurs between Left and Right components for a given value of $(j)$, namely for the same would be $\mathrm{SU}(2)_{j}$ frame.

For instance, by setting for simplicity for $B=0$ but for generic metric, we find

$$
\begin{aligned}
& f_{+-\overline{0}}(1)(G) \propto k_{R}(1)=\sqrt{2}\left[G_{11}+\frac{G_{22}}{\operatorname{det}(G)}-2\right]^{\frac{1}{2}} \\
& f_{+-\overline{0}}(2)(G) \propto k_{R}(2)=\sqrt{2}\left[G_{11}+\frac{G_{11}}{\operatorname{det}(G)}-2\right]^{\frac{1}{2}}
\end{aligned}
$$

which generalizes the expression (1.11) found for the circle. By inserting these constants into the generic DFT action it is possible to check, as sketched in the introduction, that the action for a generic spontaneous symmetry breaking to $\mathrm{U}(1)^{4}$ is achieved. The complete computation was performed by using a computer program.

The masses of the Left-vectors bosons are

$$
\begin{aligned}
& m_{1}^{2}=f_{+-0}^{2}(1)(G) \\
& m_{2}^{2}=f_{+-0}^{2}(2)(G)
\end{aligned}
$$

and (similarly for the R-vectors). They coincide with the masses computed from string theory (A.2). The values $G_{12}=0, G_{11}=G_{22}=1$ lead to $m_{1}^{2}=m_{2}^{2}=0$ thus leading to the $\mathrm{SU}(2)^{4}$ enhancing. Also, $G_{12}=0, G_{11}=1, G_{22}=\left(\frac{R_{(2)}}{\sqrt{\alpha^{\prime}}}\right)^{2}$ corresponds to a partial breaking stage to $\mathrm{SU}(2)_{1 L} \times \mathrm{U}(1)_{2 L} \times \mathrm{SU}(2)_{R} \times \mathrm{U}(1)_{2 R}$ etc.

Recall that, generically, for a given point of enhancing $\left(g_{0}, B_{0}\right)$ with $G_{L} \times G_{R}$ gauge group, once the values of fluxes $f_{A B C}(g, B)$ are found, we just have to plug them into the 
DFT action to obtain the effective gauge symmetry broken action. We have shown how to compute these fluxes from a generalized tangent frame construction. However, we can easily read them from string theory 3 -vector bosons amplitudes, as we saw for the circle case. Namely, at a given fixed point, as mentioned $k_{L}^{(\mathbb{P})}\left(g_{0}, B_{0}\right)=\alpha^{(\mathbb{P})}\left(g_{0}, B_{0}\right)$ become simple roots of the $G_{L}$ group algebra and $K_{L(R)}^{(0)}=0$ for Cartan vectors. Let us consider the 3-point amplitudes for massless bosons. For charged bosons we can write, up to an antisymmetric factor in vertex indices depending on vector polarizations (see (2.35)), as

$$
\left\langle V\left(k_{L}^{\left(\mathbb{P}_{1}\right)}\right) V\left(k_{L}^{\left(\mathbb{P}_{2}\right)}\right) V\left(k_{L}^{\left(\mathbb{P}_{3}\right)}\right)\right\rangle \propto f_{\alpha^{\left(\mathbb{P}_{2}\right)} \alpha^{\left(\mathbb{P}_{2}\right)} \alpha^{\left(\mathbb{P}_{3}\right)}}(g, B)
$$

where $f_{\alpha^{\left(\mathbb{P}_{2}\right)} \alpha^{\left(\mathbb{P}_{2}\right)} \alpha^{\left(\mathbb{P}_{3}\right)}}(g, B)=1$ if $\mathbb{P}_{3}=-\mathbb{P}_{1}-\mathbb{P}_{2}$ and vanishing otherwise due to momentum conservation. The constants are antisymmetric. At the self-dual point this indicates that structure constants $f_{\alpha_{1} \alpha_{2} \alpha_{3}}$ vanish unless $\alpha_{1}+\alpha_{2}$ is a root (and similarly for Right the sector). For the same reason mixings of Left and Right indices vanish. On the other hand, by denoting by $V\left(I_{L(R)}\right)$ with $I=1, \ldots r$ the Cartan vectors, the only non vanishing amplitudes are

$$
\left\langle V\left(k_{L}^{(\mathbb{P})}\right) V\left(k_{L}^{(-\mathbb{P})}\right) V\left(I_{L(R)}\right)\right\rangle \propto k_{L(R)}^{(\mathbb{P})}(g, B)_{I}
$$

and, by identifying the amplitudes coefficients with algebra structure constants, we have

$$
\begin{array}{ll}
k_{L}^{(\mathbb{P})}(g, B)_{I_{L}}=f_{\alpha^{(\mathbb{P})} \alpha^{(-P)} I}(g, B), & k_{R}^{(\mathbb{P})}(g, B)_{I_{R}}=f_{\hat{\alpha}^{(\mathbb{P})} \hat{\alpha}^{(-P)} \hat{I}}(g, B) \\
k_{L}^{(\mathbb{P})}(g, B)_{I_{R}}=f_{\alpha^{(\mathbb{P})} \alpha^{(-P)} \hat{I}}(g, B), & k_{R}^{(\mathbb{P})}(g, B)_{I_{L}}=f_{\hat{\alpha}^{(\mathbb{P})} \hat{\alpha}^{(-P)} I^{(}}(g, B)
\end{array}
$$

where we have used hatted indices for Right generators. Thus, we propose the algebra

$$
\begin{aligned}
{\left[E_{\alpha}, E_{-\alpha}\right] } & =k_{L}^{(\alpha) I} H_{I}+k_{R}^{(\alpha) \hat{I}} \hat{H}_{\hat{I}} & {\left[\hat{E}_{\hat{\alpha}}, \hat{E}_{-\hat{\alpha}}\right] } & =k_{L}^{(\hat{\alpha}) I} H_{I}+k_{R}^{(\hat{\alpha}) I} \hat{H}_{I} \\
{\left[H_{I}, E_{\alpha}\right] } & =k_{L}^{(\alpha) I} E_{\alpha} & {\left[\hat{H}_{\hat{I}}, \hat{E}_{\hat{\alpha}}\right] } & =k_{R}^{(\hat{\alpha}) \hat{I}} \hat{E}_{\hat{\alpha}} \\
{\left[H_{I}, \hat{E}_{\hat{\alpha}}\right] } & =k_{L}^{(\hat{\alpha}) I} \hat{E}_{\hat{\alpha}} & {\left[\hat{H}_{I}, E_{\alpha}\right] } & =k_{R}^{(\alpha) I} E_{\alpha}
\end{aligned}
$$

where we have used $\alpha=\alpha^{(\mathbb{P})}$ to alleviate the notation. It is easy to show that (3.19) satisfies Jacobi identities. and therefore defines a Lie algebra.

At the self dual point (where $k_{R}^{\alpha}\left(g_{0}, B_{0}\right)=k_{L}^{\hat{\alpha}}\left(g_{0}, B_{0}\right)=0$ ) and $f_{\alpha-\alpha I}=\alpha^{I}$, (and similarly for Right sector) the algebra reduces to the gauge algebra of $G_{L} \times G_{R}$ in the Cartan-Weyl basis. For instance notice that $\left[E_{\alpha}, E_{-\alpha}\right]=\alpha^{I} H_{I}$ for charged generators $E_{\alpha}$ and Cartan generators $H_{I}$, as expected.

As an example let us specify to the $\mathrm{SU}(3)_{L} \times \mathrm{SU}(3)_{R}$ case (the expressions are, however, general). Since this algebra must be continuously connected with the $\mathrm{SU}(3)_{L} \times \mathrm{SU}(3)_{R}$ algebra at the fixed point and has four Cartan generators the only possibility left is an $\mathrm{SU}(3) \times \mathrm{SU}(3)$. Again, away from the fixed point, we detect the same underlying algebra, now mixing massive and massless (associated to Cartan generators) vector fields.

Let us underscore that, by replacing above fluxes into the DFT action (1.3) and by performing the scalars expansion (2.13), as we did for the circle case example, the full broken $G_{L} \times G_{R}$ symmetry action is found. Recall that this is valid for an arbitrary fixed point in a general $r$ dimensional toroidal compactification. As a check we show in the appendix that the resulting masses for vector fields and scalar fields, as functions of moduli, coincide with the string theory ones. 


\section{Summary and outlook}

A well known distinguished feature of string theory is the enhancing of gauge symmetries at certain values of moduli backgrounds. In this work we have shown that DFT formulation helps to identify an interesting description of enhancing phenomena. Namely, enhancing information appears encoded into moduli dependent generalized fluxes $f_{A B C}(g, B)$ with $A, B, C=1, \ldots 2 n$ indices in an $O(n, n)$ vector representation. Splitting indices in a LeftRight basis $A=(a, \hat{a})$, it appears that enhancing occurs for moduli values $\left(g_{0}, B_{0}\right)$ such that generalized fluxes with mixed indices vanish. In this situation $f_{a b c}\left(g_{0}, B_{0}\right)\left(f_{\hat{a} \hat{b} \hat{c}}\left(g_{0}, B_{0}\right)\right)$ become the structure constants of a $G_{L}\left(G_{R}\right), \operatorname{dim} G_{L}=n$ dimensional non-Abelian gauge group. In fact, the vector boson masses are proportional to mixed indices fluxes (B.3).

As mentioned, when replacing these moduli dependent fluxes into the generic DFT action the effective string theory action is reproduced, as long as up to slightly massive states are kept. Therefore, DFT is providing us with a generic field theory action that leads to an accurate description of string theory results even in a non trivial stringy situation of gauge symmetry enhancing-breaking when massive states with associated momenta and winding are present. As discussed in [3] for the circle case (and extended in [44] for other situations) by giving vev's to some specific scalar fields, the string broken symmetry action can be approximately obtained as an expansion in powers of the vev,s. It is worth insisting that the DFT construction we are presenting here is already producing the broken symmetry phase. Moreover, different coefficients and masses in the string action are exactly reproduced as functions of moduli and not as an expansion.

In addition, we have shown (at least for some examples) that generalized fluxes can be computed by introducing a generalized frame in tangent space with extended tangent directions but depending only on the coordinates of the double "physical torus". The DFT generalized Lie algebra closes even though the strong constraint is not satisfied. In fact, the frame is explicitly non-geometric since it is a function of the double coordinates $\mathbb{Y}=(Y, \tilde{Y})$.

The idea of doubling the number of coordinates in order to describe winding modes was one of the original motivations of DFT. However, only recently windings were actually included in DFT. In [3] a step in this direction was performed by showing that DFT can describe the massless sector of an enhanced gauge symmetry situation with windings playing a fundamental role and where an unpaired number of Left and Right $N-\bar{N}= \pm 1$ moving oscillators is implied in string theory (see also [44]). Also in [34] a generalized KK toroidal compactification (GKK) of DFT containing towers of massive states with generic windings and KK momenta was considered, for the case $N-\bar{N}=0$, namely with the level matching condition $\mathbb{P}^{2}=0$. The present work is a contribution in between, in the sense that it incorporates slightly massive states with paired and unpaired oscillators but disregards higher massive states.

The tangent space extra dimensions in the above construction are associated to states with non vanishing momenta and windings, actually with $\mathbb{P}^{2}= \pm 1$. It may appear somewhat awkward that moving continuously from one point of enhancing to another could lead to a discrete change in the number of these extra tangent dimensions, even if these are just tangent directions and not physical dimensions at all. In string theory the vector 
fields that become massless to lead to gauge enhancing are part of the spectrum and they are associated to $N-\bar{N}= \pm 1$. It appears that in this situation DFT in lower dimensions should allow for the presence of new vector fields, say $A_{L(R)}^{\nu}(x, \mathbb{Y})$ where $\mathbb{Y}$ are coordinates on a double torus.

A possible way these jumps could be actually understood is through a GKK mode expansion, as considered in [34], but allowing for states with $\operatorname{LMC} \delta\left(\mathbb{P}^{2}\right)= \pm 1,0$. For instance,

$$
\begin{aligned}
A_{L \nu}(x, \mathbb{Y}) & =\sum_{\mathbb{P}} A_{L \nu}^{(\mathbb{P})}(x) e^{i \mathbb{P}_{M} \mathbb{Y}^{M}} \delta\left(\mathbb{P}^{2}, 1\right) \\
& =\sum_{\mathbb{P}} A_{L \nu}^{I(\mathbb{P})}(x) e^{i k_{L} \cdot y_{L}+k_{R} \cdot y_{R}} \delta\left(\mathbb{P}^{2}, 1\right),
\end{aligned}
$$

where $P_{L}, P_{R}$ depend on moduli (3.3). When moving continuously along the moduli space, for certain values of $\mathbb{P}$, GKK modes $k_{R}=0$ and the corresponding fields $A_{L \nu}^{(\mathbb{P})}(x)$ become massless. For instance for the $T^{2} \times \tilde{T}^{2}$ the six modes (3.6) become massless for $g_{11}=$ $g_{22}=-2 g_{12}=-2 B_{12}=1$ leading to the charged operators of $\mathrm{SU}(3)_{L}$. Sliding away from this point the masses of these modes vary continuously from zero. When reaching the moduli point $g_{11}=g_{22}=1 ; B_{12}=0$ other modes (the six modes shown in (3.11)) become massless ${ }^{9}$ and lead to $\mathrm{SU}(2)_{L}^{2}$ enhancing. The massless vector fields are those captured by the extended tangent frame vector in DFT. Moreover, we saw that at the neighbourhood of the point of enhancing associated to a gauge generator algebra $G$, there is still an underlying global $G$ algebra, mixing massless (Cartans) and slightly massive states. When moving away from that point other fields, now with comparable masses, will come into play and will have non neglectable 3-point amplitudes indicating a possible infinite enhancing of the global algebra. This appears to be an indication of the presence of a Generalized Kac-Moody algebra of the kind discussed in [34] but including unpaired LMC conditions. Of course these ideas need further investigation.

For the sake of simplicity we have dealt with the bosonic string example. However the reasoning should be straightforwardly applicable to the (bosonic sector) of Heterotic theories ([21]) or Type II theories obtained from U-dual Extended Field Theories (EFT) [42, 43]. It could also be interesting to explore the inclusion of extra tangent dimensions directly in gauged supergravity theories [31, 32].

Note added in proof. The same day this paper was made public, the article [44] appeared adressing similar issues from a rather complementary point of view.

\section{Acknowledgments}

We thank G. Torroba, F. Schaposnik Massolo and D. Marqués for useful discussions and comments and, in particular, C. Nuñez who participated in the first steps of this research. This work was supported by PIP CONICET grant, PICT-2012-513 and by IBS-R018-D2. G. A. thanks the Instituto de Física Teórica (IFT UAM-CSIC) in Madrid for its support

\footnotetext{
${ }^{9}$ Notice that there are two common modes $\mathbb{P}=( \pm 1,0, \pm 1,0)$.
} 
via the Centro de Excelencia Severo Ochoa Program under Grant SEV-2012-0249 and A.S.ICTP for hospitality and partial support during the first steps of this work.

\section{A Vertex operators and enhancing}

We summarize here some string theory ingredients needed in the body of the article.

A generic vertex operator contains an exponential contribution that can be written in terms of Left and Right moving coordinates $y_{L}(z), y_{R}(\bar{z})$ as $e^{i k \cdot X+i k_{L} \cdot y_{L}+i k_{R} \cdot \bar{y}_{R}}$ : where $K^{\mu}$ stands for the space-time momentum while $k_{L(R)}$ are the internal $L(R)$ momenta. It is convenient to use coordinates $y_{L(R)}^{a}=e_{m}{ }^{a} y_{L(R)}^{m}$ with tangent space indices $a, b, \ldots$, defined in terms of the vielbein $e_{m}{ }^{a}\left(\delta^{a b}=e_{m}{ }^{a} g^{m n} e_{n}{ }^{b}\right)$ since they have the standard OPEs. Namely, the propagators read

$$
\begin{aligned}
\left\langle X^{\mu}(z, \bar{z}) X^{\nu}(w, \bar{w})\right\rangle & =-\frac{\alpha^{\prime}}{2} \eta^{\mu \nu} \ln |z-w|^{2}, \\
\left\langle Y^{a}(z) Y^{b}(w)\right\rangle & =-\delta^{a b} \frac{\alpha^{\prime}}{2} \ln (z-w), \quad\left\langle\bar{Y}^{a}(\bar{z}) \bar{Y}^{b}(\bar{w})\right\rangle=-\delta^{a b} \frac{\alpha^{\prime}}{2} \ln (\bar{z}-\bar{w}) .
\end{aligned}
$$

and the vertex operator momenta are

$$
k_{L}^{a}=e^{a}{ }_{m} p_{L}^{m}, \quad k_{R}^{a}=e^{a}{ }_{m} p_{R}^{m}
$$

where

$$
p_{L}^{m}=\tilde{p}^{m}+g^{m n}\left(p_{n}-B_{n k} \tilde{p}^{k}\right), \quad p_{R}^{m}=-\tilde{p}^{m}+g^{m n}\left(p_{n}-B_{n k} \tilde{p}^{k}\right) .
$$

The stress energy tensor is

$$
T(z)=-\frac{1}{\alpha^{\prime}}\left(\eta_{\mu \nu}: \partial_{z} X^{\mu}(z) \partial_{z} X^{\nu}(z):+\delta_{a b}: \partial_{z} Y^{a}(z) \partial_{z} Y^{b}(z):\right),
$$

The mass of the string states is

$$
M^{2}=\frac{1}{2} m_{L}^{2}+\frac{1}{2} m_{R}^{2}=\frac{1}{2} k_{a L} \cdot k_{a L}+\frac{1}{2} k_{a R} \cdot k_{a R}+2(N+\bar{N}-2)
$$

where $N, \bar{N}$ are the number of string oscillators and the level matching condition reads

$$
\frac{1}{4} k_{a L} \cdot k_{a L}-\frac{1}{4} k_{a R} \cdot k_{a R}-(N-\bar{N})=p_{n} \tilde{p}^{n}-(N-\bar{N})=0
$$

and similarly for the right moving one.

\section{A.1 Torus example}

The frame base can be written as (as mention the factor $\sqrt{2}$ is included to maintain the normalization conditions $\alpha^{2}=2$ for simple roots)

$$
e_{1}=\frac{1}{\sqrt{2}}\left(0, \sqrt{G_{11}}\right), \quad e_{2}=\frac{1}{\sqrt{2}}\left(\frac{\sqrt{\operatorname{det} G}}{\sqrt{G_{11}}}, \frac{G_{12}}{\sqrt{G_{11}}}\right)
$$


leading to the matrix $g_{m n}=e_{m} \cdot e_{n}=\frac{1}{2} G_{m n}$. with dual lattice vectors $\left(e_{m}^{*}=e^{m}\right)$

$$
e_{1}^{*}=\sqrt{2}\left(-\frac{G_{12}}{\sqrt{\operatorname{det} G} \sqrt{G_{11}}}, \frac{1}{\sqrt{G_{11}}}\right), \quad e_{2}^{*}=\sqrt{2}\left(\frac{\sqrt{G}_{11}}{\sqrt{\operatorname{det} G}}, 0\right)
$$

A field $B=B_{12}\left(\begin{array}{ll}0 & 1 \\ 1 & 0\end{array}\right)$ can also be introduced. When

$$
G=\left(\begin{array}{cc}
2 & -1 \\
-1 & 2
\end{array}\right)
$$

the $\mathrm{SU}(3)$ Cartan matrix is obtained and frame vectors become $e_{m}=\frac{1}{\sqrt{2}} \alpha_{m}$ where

$$
\alpha_{1}=(0, \sqrt{2}), \quad \alpha_{2}=\left(\frac{\sqrt{3}}{\sqrt{2}},-\frac{1}{\sqrt{2}}\right)
$$

are the $\mathrm{SU}(3)$ simple roots.

On the other hand, $G_{22}=G_{11}=2 ; G_{12}=0$ corresponds to an $\mathrm{SU}(2) \times \mathrm{SU}(2)$ algebra.

Metric and $B$ field define the complex structure $U=U_{1}+i U_{2}$ and Khaler structure $T=T_{1}+i T_{2}$ of the torus with $U_{1}=\frac{g_{12}}{g_{22}}, U_{2}=\frac{\sqrt{\operatorname{det} g}}{g_{22}}, T_{1}=B_{12}, T_{2}=\sqrt{\operatorname{det} g}$. In terms of complex moduli, $\mathrm{SU}(3)_{L} \times \mathrm{SU}(3)_{R}$ enhancing occurs at

$$
T=-\frac{1}{2}+i \frac{\sqrt{3}}{2}=U
$$

whereas $(\mathrm{SU}(2) \times \mathrm{SU}(2))_{L} \times(\mathrm{SU}(2) \times \mathrm{SU}(2))_{R}$ enhancing is achieved for

$$
T=i=U
$$

\section{B General enhancing groups}

We show here that, in the general case of an enhancing from $\mathrm{U}(1)_{L}^{r} \times \mathrm{U}(1)_{R}^{r}$ to a gauge group $G_{L} \times G_{R}$ the generalized fluxes lead to the exact vector and scalar massive terms. Namely, the corresponding masses coincide with the masses computed from string theory. Consider the L-R splitting of indices in the $\mathcal{C}$ base $A=(a, \hat{a})$ where the first (second) entries belong to left group $G_{L}$ (right group $G_{R}$ ). Let us focus on $G_{L}$ and further split left indices as $a=(\alpha, I)$ corresponding to charged generators and Cartan generators $I=1, \ldots r$ (and similarly for Right group).

\section{B.1 Vector masses}

The vector mass terms in the Lagrangian read

$$
\begin{aligned}
\left(f_{A B C} A_{\mu}^{B} M_{D}^{C}+f_{D B C} A_{\mu}^{B} M_{A}^{C}\right)^{2} & \sim A_{\mu}^{B} A^{E \mu} f_{A B C} f_{D E F}\left(\eta^{A D} \eta^{C F}-\delta^{A D} \delta^{C F}\right) \\
& \sim A_{\mu}^{B} A^{E \mu} f_{a B \hat{c}} f_{a E \hat{c}}
\end{aligned}
$$


If the fluxes do not mix Left and Right sectors (as it happens at the self dual point) then all vectors are massless. From momentum conservation we know that $f_{a I \hat{c}}=f_{a \bar{I} \hat{c}}=0$. Moreover $a$ and $\hat{c}$ can not be charged indices simultaneously. Then

$$
f_{a B \hat{c}} f_{a E \hat{c}}=f_{I B \hat{\gamma}} f_{I E \hat{\gamma}}+f_{\alpha B \hat{I}} f_{\alpha E \hat{I}}
$$

We conclude that indices $B, E$ in the previous expression must be charged indices and, moreover, they must be equal by momentum conservation

$$
\begin{aligned}
A_{\mu}^{B} A^{E \mu} f_{a B \hat{c}} f_{a E \hat{c}} & \sim \sum_{\hat{\gamma}} A_{\mu}^{\hat{\gamma}} A^{\hat{\gamma} \mu} \sum_{I=1}^{r} f_{I-\hat{\gamma} \hat{\gamma}} f_{I-\hat{\gamma} \hat{\gamma}}+\sum_{\alpha} A_{\mu}^{\alpha} A^{\alpha \mu} \sum_{\hat{I}=1}^{r} f_{\alpha-\alpha \hat{I}} f_{\alpha-\alpha \hat{I}} \\
& =\sum_{\hat{\gamma}} A_{\mu}^{\hat{\gamma}} A^{\hat{\gamma} \mu} m_{\hat{\gamma}}^{2}+\sum_{\alpha} A_{\mu}^{\alpha} A^{\alpha \mu} m_{\alpha}^{2}
\end{aligned}
$$

where the sum runs over the positive roots. By using that (see (3)) $f_{I-\hat{\gamma} \hat{\gamma}}=K_{L, \hat{\gamma}}^{I}$ i.e. the I-component of the Left $\gamma$ momentum (similar for the right case) we can write the masses as $m_{\hat{\gamma}}^{2}=\sum_{I=1}^{r}\left(K_{L, \hat{\gamma}}^{I}\right)^{2}$ and for the $\gamma$-left vector is $m_{\gamma}^{2}=\sum_{I=1}^{r}\left(K_{R, \hat{\gamma}}^{I}\right)^{2}$, that coincide with vector masses computed from (A.2).

\section{B.2 Scalar masses}

We denote the, $(\operatorname{dim} G-r)^{2}$, massless scalars charged under Left and Right gauge group as $M^{\alpha \hat{\beta}}$. In string compactification they are described by the vertex operators $V^{\alpha \hat{\beta}}(z, \bar{z}) \propto$ $J^{\alpha}(z) \hat{J}^{\hat{\beta}}(\bar{z})$ with $J^{\alpha}(z)=e^{k_{L \alpha} \cdot y}$. When moving away from the self-dual point a non vanishing Right contribution $k_{R \alpha}\left(m_{-}\right.$in circle example) appears and similarly a $k_{L \hat{\beta}}$, from the Right sector. Therefore, the scalar Left and Right internal momenta become

$$
\begin{aligned}
& k_{L \alpha \hat{\beta}}=k_{L \alpha}+k_{L \hat{\beta}} \\
& k_{R \hat{\beta} \alpha}=k_{R \hat{\beta}}+k_{R \alpha}
\end{aligned}
$$

Recall that, since $N=\bar{N}=0$ level matching requires $k_{L \alpha \hat{\beta}}^{2}=k_{R \hat{\beta} \alpha}^{2}$.

The mass of the scalar is (A.2)

$$
M_{\alpha \hat{\beta}}^{2}=\frac{1}{2} k_{L \alpha \hat{\beta}}^{2}+\frac{1}{2} k_{R \hat{\beta} \alpha}^{2}-4
$$

By replacing the values (B.4) into this formula and by using LMC for vector currents

$$
\begin{aligned}
& k_{L \alpha}^{2}-k_{R \alpha}^{2}=1 \\
& k_{L \hat{\beta}}^{2}-k_{R \hat{\beta}}^{2}=-1
\end{aligned}
$$

we obtain

$$
M_{\alpha \hat{\beta}}^{2}=k_{R \alpha} \cdot\left(k_{R \alpha}+k_{R \hat{\beta}}\right)+k_{L \hat{\beta}} \cdot\left(k_{L \hat{\beta}}+k_{L \alpha}\right)
$$

that, as expected, vanishes at the fixed point. By using the identification with fluxes (3) this expression can be recast as

$$
M_{\alpha \hat{\beta}}^{2}=f_{\hat{I} \alpha-\alpha}\left(f_{\hat{I} \alpha-\alpha}+f_{\hat{I} \hat{\beta}-\hat{\beta}}\right)+f_{I \hat{\beta}-\hat{\beta}}\left(f_{I \hat{\beta}-\hat{\beta}}+f_{I \alpha-\alpha}\right)
$$


This is exactly the combination of fluxes that appears in front of the quadratic scalar term when we mimmick the steps we followed for the circle case (2.30). Namely, insert the expansion in scalar fluctuations $M$ (2.13), into the third row of the DFT action (1.3) and use the values (1.11).

Open Access. This article is distributed under the terms of the Creative Commons Attribution License (CC-BY 4.0), which permits any use, distribution and reproduction in any medium, provided the original author(s) and source are credited.

\section{References}

[1] K.S. Narain, New heterotic string theories in uncompactified dimensions $<10$, Phys. Lett. B 169 (1986) 41 [INSPIRE].

[2] A. Giveon, M. Porrati and E. Rabinovici, Target space duality in string theory, Phys. Rept. 244 (1994) 77 [hep-th/9401139] [INSPIRE].

[3] G. Aldazabal, M. Graña, S. Iguri, M. Mayo, C. Núñez and J.A. Rosabal, Enhanced gauge symmetry and winding modes in Double Field Theory, JHEP 03 (2016) 093 [arXiv: 1510.07644] [INSPIRE].

[4] G. Aldazabal, D. Marqués and C. Núñez, Double Field Theory: a pedagogical review, Class. Quant. Grav. 30 (2013) 163001 [arXiv:1305.1907] [INSPIRE].

[5] G. Aldazabal, W. Baron, D. Marqués and C. Núñez, The effective action of Double Field Theory, JHEP 11 (2011) 052 [Erratum ibid. 11 (2011) 109] [arXiv:1109.0290] [INSPIRE].

[6] D. Geissbuhler, Double Field Theory and $N=4$ gauged supergravity, JHEP 11 (2011) 116 [arXiv: 1109.4280] [INSPIRE].

[7] D. Geissbuhler, D. Marqués, C. Núñez and V. Penas, Exploring Double Field Theory, JHEP 06 (2013) 101 [arXiv: 1304.1472] [INSPIRE].

[8] N. Hitchin, Generalized Calabi-Yau manifolds, Quart. J. Math. 54 (2003) 281 [math/0209099] [INSPIRE].

[9] M. Gualtieri, Generalized complex geometry, DPhil thesis, Oxford University, Oxford U.K., (2004) [math/0401221] [INSPIRE].

[10] M. Graña, R. Minasian, M. Petrini and D. Waldram, T-duality, generalized geometry and non-geometric backgrounds, JHEP 04 (2009) 075 [arXiv:0807.4527] [INSPIRE].

[11] A. Coimbra, C. Strickland-Constable and D. Waldram, Supergravity as generalised geometry I: type II theories, JHEP 11 (2011) 091 [arXiv:1107.1733] [INSPIRE].

[12] C. Hull and B. Zwiebach, Double Field Theory, JHEP 09 (2009) 099 [arXiv:0904.4664] [INSPIRE].

[13] O. Hohm, C. Hull and B. Zwiebach, Background independent action for Double Field Theory, JHEP 07 (2010) 016 [arXiv: 1003.5027] [INSPIRE].

[14] O. Hohm, C. Hull and B. Zwiebach, Generalized metric formulation of Double Field Theory, JHEP 08 (2010) 008 [arXiv: 1006.4823] [inSPIRE].

[15] M.J. Duff, Duality rotations in string theory, Nucl. Phys. B 335 (1990) 610 [InSPIRE]. 
[16] M.J. Duff and J.X. Lu, Duality rotations in membrane theory, Nucl. Phys. B 347 (1990) 394 [INSPIRE].

[17] A.A. Tseytlin, Duality symmetric formulation of string world sheet dynamics, Phys. Lett. B 242 (1990) 163 [inSPIRE].

[18] A.A. Tseytlin, Duality symmetric closed string theory and interacting chiral scalars, Nucl. Phys. B 350 (1991) 395 [inSPIRE].

[19] W. Siegel, Superspace duality in low-energy superstrings, Phys. Rev. D 48 (1993) 2826 [hep-th/9305073] [INSPIRE].

[20] W. Siegel, Two vierbein formalism for string inspired axionic gravity, Phys. Rev. D 47 (1993) 5453 [hep-th/9302036] [INSPIRE].

[21] O. Hohm and S.K. Kwak, Double Field Theory formulation of heterotic strings, JHEP 06 (2011) 096 [arXiv:1103.2136] [INSPIRE].

[22] I. Jeon, K. Lee and J.-H. Park, Stringy differential geometry, beyond Riemann, Phys. Rev. D 84 (2011) 044022 [arXiv: 1105.6294] [INSPIRE].

[23] D.S. Berman and D.C. Thompson, Duality symmetric string and M-theory, Phys. Rept. 566 (2014) 1 [arXiv:1306.2643] [INSPIRE].

[24] O. Hohm, D. Lüst and B. Zwiebach, The spacetime of Double Field Theory: review, remarks and outlook, Fortsch. Phys. 61 (2013) 926 [arXiv:1309.2977] [INSPIRE].

[25] O. Hohm, W. Siegel and B. Zwiebach, Doubled $\alpha^{\prime}$-geometry, JHEP 02 (2014) 065 [arXiv:1306.2970] [INSPIRE].

[26] O. Hohm and B. Zwiebach, Double Field Theory at order $\alpha^{\prime}$, JHEP 11 (2014) 075 [arXiv: 1407.3803] [INSPIRE].

[27] O.A. Bedoya, D. Marqués and C. Núñez, Heterotic $\alpha^{\prime}$-corrections in Double Field Theory, JHEP 12 (2014) 074 [arXiv: 1407.0365] [INSPIRE].

[28] D. Marqués and C.A. Núñez, T-duality and $\alpha^{\prime}$-corrections, JHEP 10 (2015) 084 [arXiv: 1507.00652] [INSPIRE].

[29] A. Coimbra, R. Minasian, H. Triendl and D. Waldram, Generalised geometry for string corrections, JHEP 11 (2014) 160 [arXiv:1407.7542] [INSPIRE].

[30] J. Scherk and J.H. Schwarz, How to get masses from extra dimensions, Nucl. Phys. B 153 (1979) 61 [inSPIRE].

[31] H. Samtleben, Lectures on gauged supergravity and flux compactifications, Class. Quant. Grav. 25 (2008) 214002 [arXiv:0808.4076] [INSPIRE].

[32] M. Trigiante, Gauged supergravities, Phys. Rept. 680 (2017) 1 [arXiv:1609.09745] [INSPIRE].

[33] M. Graña and D. Marqués, Gauged Double Field Theory, JHEP 04 (2012) 020 [arXiv: 1201.2924] [INSPIRE].

[34] G. Aldazabal, M. Mayo and C. Núñez, Probing the string winding sector, JHEP 03 (2017) 096 [arXiv: 1611.04927] [INSPIRE].

[35] G. Dibitetto, J.J. Fernandez-Melgarejo, D. Marqués and D. Roest, Duality orbits of non-geometric fluxes, Fortsch. Phys. 60 (2012) 1123 [arXiv:1203.6562] [INSPIRE]. 
[36] K. Lee, C. Strickland-Constable and D. Waldram, New gaugings and non-geometry, arXiv: 1506.03457 [INSPIRE].

[37] R. Blumenhagen, F. Hassler and D. Lüst, Double Field Theory on group manifolds, JHEP 02 (2015) 001 [arXiv: 1410.6374] [INSPIRE].

[38] M.B. Schulz, T-folds, doubled geometry and the SU(2) WZW model, JHEP 06 (2012) 158 [arXiv: 1106.6291] [INSPIRE].

[39] K. Lee, C. Strickland-Constable and D. Waldram, Spheres, generalised parallelisability and consistent truncations, arXiv:1401.3360 [INSPIRE].

[40] G. Dall'Agata, G. Inverso and M. Trigiante, Evidence for a family of $\mathrm{SO}(8)$ gauged supergravity theories, Phys. Rev. Lett. 109 (2012) 201301 [arXiv:1209.0760] [INSPIRE].

[41] O. Hohm and S.K. Kwak, Double Field Theory formulation of heterotic strings, JHEP 06 (2011) 096 [arXiv:1103.2136] [INSPIRE].

[42] O. Hohm and H. Samtleben, Gauge theory of Kaluza-Klein and winding modes, Phys. Rev. D 88 (2013) 085005 [arXiv: 1307.0039] [INSPIRE].

[43] G. Aldazabal, M. Graña, D. Marqués and J.A. Rosabal, The gauge structure of exceptional field theories and the tensor hierarchy, JHEP 04 (2014) 049 [arXiv: 1312.4549] [INSPIRE].

[44] Y. Cagnacci, M. Graña, S. Iguri and C. Núñez, The bosonic string on string-size tori from Double Field Theory, JHEP 06 (2017) 005 [arXiv:1704.04242] [INSPIRE]. 